\title{
Changes in Employment Status after Myocardial Infarction among Men
}

\author{
Ceyda Şahan ${ }^{1}$, Yücel Demiral ${ }^{1}$, Bülent Kılıç ${ }^{1}$, Özgür Aslan ${ }^{2}$ \\ ${ }^{1}$ Department of Public Health, Dokuz Eylül University School of Medicine, İzmir, Turkey \\ ${ }^{2}$ Department of Cardiology, Dokuz Eylül University School of Medicine, İzmir, Turkey
}

Background: According to the Turkey Burden of Disease Study, $10 \%$ of the national burden of disease is attributed to cardiovascular diseases. Although the standardized coronary heart disease (CHD) rate is falling in general, CHD prevalence among young people is rising. On the other hand, as a result of increased life expectancy and higher retirement ages, the CHD rate among workers is also increasing. Therefore, work ability and return to work after diagnosis are important for population health and well-being. Socioeconomic factors and working conditions may play a key role as well as clinical conditions described in the literature that affect returning to work.

Aims: The aims of this qualitative study are exploring the changes in employment and working conditions of the patients after acute myocardial infarction (AMI) and affecting factors such as socioeconomic, personal and environmental. Study design: Qualitative research.

Methods: The research population are fifty-three patients who are engaged in paid employment when the people have been diagnosed with myocardial infarction for the first time between 2011 and 2012 at a university hospital coronary care unit. We intended to reach the whole population. Twen- ty-seven patients were contacted whose phone numbers were accessible from the hospital records. Semi-structured in-depth interviews were conducted with twelve patients in a meeting room at the hospital. The interviews were tape-recorded accompanied by note-taking and the content analysis method were evaluated.

Results: While many of the participants continued to work at the same job by working less, one third of them said that they were thinking about getting an easier job if they have the opportunity. On the other hand, in most cases, there were neither assessments about their work ability, nor changes to their working conditions after AMI. They had to cope with their conditions, such as economic or psychosocial, without any support.

Conclusions: While the patients have to return to work for economic and social reasons, they expressed uncertainty about working after a diagnosis of AMI and could not reach professional support to assess their work abilities. Therefore, specific algorithms and assessment tools to manage the return to work of AMI patients would be useful.

Keywords: Employment, myocardial infarction, return to work, working conditions
According to the Turkey Burden of Disease Study, coronary heart disease (CHD) is one of the three diseases with the highest mortality, and $10 \%$ of the national burden of disease is attributed to cardiovascular diseases (1). Although the standardized CHD rate is falling in general, CHD prevalence among young people is rising (2). On the other hand, as a result of increased life expectancy and higher retirement ages, the CHD rate of workers is also increasing day by day (3). People of working age who suffer from chronic disease are not considered employable. In particular, chronic patients who provide for themselves have low incomes and have to cope with poverty. This situation creates an economic burden for both patients and their families (4).

Although some CHD patients undergo coronary angiography or coronary bypass surgery, some are monitored by medical treatment only. Some patients have other accompanying chronic diseases and have to take at least 3 medications. This may negatively affect their work lives and their quality of life (5). Some factors described in the literature that may affect the

This study was presented at the $31^{\text {st }}$ International Congress on Occupational Health, 31 May-5 June 2015, Seoul, Korea.

Address for Correspondence: Dr. Ceyda Şahan, Department of Public Health, Dokuz Eylül University School of Medicine, İzmir, Turkey

Phone: +905054332282 e-mail: ceyda_sahan@hotmail.com

Received: 27 April 2015 Accepted: 1 September 2015

• DOI: 10.5152/balkanmedj.2016.150611

Available at www.balkanmedicaljournal.org

Cite this article as:

Şahan C, Demiral Y, Kılıç B, Aslan Ö. Changes in employment status after myocardial infarction among men. Balkan Med J 2016;33:419-25 
return to work are complications related to disease, but socioeconomic factors play a role as well. Studies have found that people with left ventricular dysfunction and low job satisfaction are less likely to return to work in particular $(6,7)$. A study conducted in 1990 in the United States found that older people with coronary heart disease who are mentally weak and physically incompetent, black, insufficiently educated, have suffered from congestive heart failure and extracardiac vascular disease, and belong to a low occupational class are more likely to leave the labor force (8). According to a qualitative study, favorable working conditions, work relationships, social environment, and economic status affect whether people return to work (9). There are no studies that analyze how coronary heart disease affects the working lives of patients and whether they return to work in Turkey. The studies in this area would provide important knowledge about conditions of CHD patients after their diagnosis. Since there are lots of factors such as social, economic, clinical aspects, working conditions that may affect return to work after diagnosis, qualitative research methodology is preferred to understand the multidimensional structure of the problem.

The aims of this qualitative study are to explore the changes in employment and working conditions of the patients after AMI and affecting factors such as socioeconomic, personal and environmental.

\section{MATERIALS AND METHODS}

This is a qualitative study. This research aimed to interview with the patients who were diagnosed with AMI in a university hospital for the first time between 31 December 2011 and 2012. These patients were working in a paid job when they suffered from AMI, and were between 18 and 50 years old. The records showed that there were 53 patients who met these criteria. Of the 53 patients, 4 were female, and 53 were male. We obtained the phone numbers of 27 patients and called them. The 12 patients who agreed to participate in our study were interviewed using a semi-structured questionnaire. All patients were invited to the hospital and interviews were carried out in a meeting room by a researcher. The average interview time was 34.5 (15-57) minutes. Since none of the women could be reached, they were not interviewed. The Local Ethical Committee has reviewed and approved the study protocol. Verbal and written consent was obtained from all participants.

The participants were asked for their name, age, marital status, educational status, occupation, job before diagnosis, current working status, job after diagnosis, number of children and job status, as well as whether they have parents or children in need of care. Participants were interviewed using a semi-structured interview guide.
The interviews were recorded, transcribed and evaluated using content analysis. Content analysis was conducted manually. For statistical analyses, Excel 2007 (Microsoft Inc.; Redmond, Washington, United States of America) was used. The texts were encoded separately by three researchers and then a common list of codes was created to identify the primary and secondary themes. The primary themes were identified as quantitative job change, qualitative job change, and the idea of quantitative job change. These themes were configured in accordance with job insecurity literature. While quantitative job insecurity was described as worries about losing the job itself, qualitative job insecurity was explained as worries about losing important job features such as deterioration of working conditions, demotion, lack of career opportunities, decreasing salary development (10). Quantitative job change describes the patient taking another job after the AMI, being retired or quitting his job. Qualitative job change on the other hand describes the patient continuing to work at the same job, but with changed duties or amounts of work. The idea of quantitative job change means that the patient thinks about finding an easier job after the AMI. Other reasons affecting the working status include continuing to work for economic reasons, working because of gender role, limitations in the social environment, mental problems, feeling physically incompetent, work stress and doctors' views about the patients working.

\section{RESULTS}

All of the participants were male, and their average age was 43.9 (32-49). Before their AMI diagnosis, 1 of the participants were working in the public sector, 8 were working for private companies, and 3 of them owned their own business (Table 1). While coronary angiography treatment was applied to all participants, none of them have undergone bypass surgery. The average number of medicines used by patients was 6.5 (5-8). While 5 patients had mild to moderate heart failure, only one patient had another chronic disease without CHD. During the interviews, while 9 patients were still working at the same job, 1 had been dismissed after the AMI. Two patients started another job voluntarily. While many of the participants continued to work at the same job by working less, one third of them said that they were thinking about getting an easier job if they were given the opportunity.

It is been found that while quantitative job change was low, qualitative job change was quite high. Most of the patients started to work for less at the same job after AMI. One patient said, "I do not care about my work anymore. When I am bored, I am getting out. It depends on my pleasure. I am spending more time with my family than before. For example, 
TABLE 1. Descriptive characteristics of participants

\begin{tabular}{lccccc}
\hline Patient Number & Age & Marital Status & Education & Family Members in Need of Care & Job before Diagnosis \\
\hline 1 & 40 & Married & High school & Yes & Financial advisor \\
2 & 49 & Married & High school & Yes & Officer of the municipality \\
3 & 48 & Married & Secondary school & Yes & Plumber in a gas station \\
4 & 45 & Married & Primary school & Yes & Garden arrangements \\
5 & 45 & Divorced & High school & No & Worker in a shopping center \\
6 & 32 & Married & High school & No & Driver \\
7 & 49 & Married & High school & No & Worker in a restaurant \\
8 & 38 & Married & Secondary school & No & Yes \\
9 & 49 & Divorced & High school & Yes & Worker in the private sector \\
10 & 42 & Married & Secondary school & Yes & Building contractor \\
11 & 49 & Married & High school & Worker in a company \\
12 & 41 & Married & Secondary school & Winess \\
\hline
\end{tabular}

I felt embarrassed yesterday and I didn't go to work, despite it being necessary". Also, some patients had the idea of quantitative job change. A patient explained this idea as: "If I find more relax and less tired one, I would like to change my job. But it seems that difficult to find more comfortable job". On the other hand, in most of the cases, there was neither assessment about work ability, nor arrangements in working conditions after AMI. They had to cope with their economic or psychosocial problems without any professional support.

There are many reasons which affect returning to work after AMI. We showed these relationships in Figure 1. The reasons were grouped as facilitators and barriers. While economic reasons and gender role may be facilitators of returning to work with qualitative job change, limitations in the social environment, mental problems, work stress and feeling physically incompetent may cause quantitative job changes such as quitting their job, retiring or taking another job. Also, doctors' views about the patient working may affect the idea of quantitative job change, or quantitative job change directly.

\section{Facilitators of returning to work after AMI}

Most of the people who continued to work in the same job stated that they did not quit because they had to look after their family and lacked economic means. A patient explained: "I didn't work for two months when I was in hospital. Because of the rent money, electricity and water bills, all of my savings ran out, and I got into debt. If I do not work, we will be out of money. If I do not work, we will starve. So I must work". One patient said that even if he retired, his income would not be sufficient. Therefore, he would still have to continue to work.

The patients stated that as males, they feel obliged to earn money after their illness, and that if they do not work their family will have economic problems. Moreover, five patients said that they do not want to take advantage of the money their wives earn because of gender oppression. According to a patient "Looking after children economically is the duty of the male as Turkish traditions suggest. Even if my wife goes to work, I'm not the type of person to spend the money she earns".

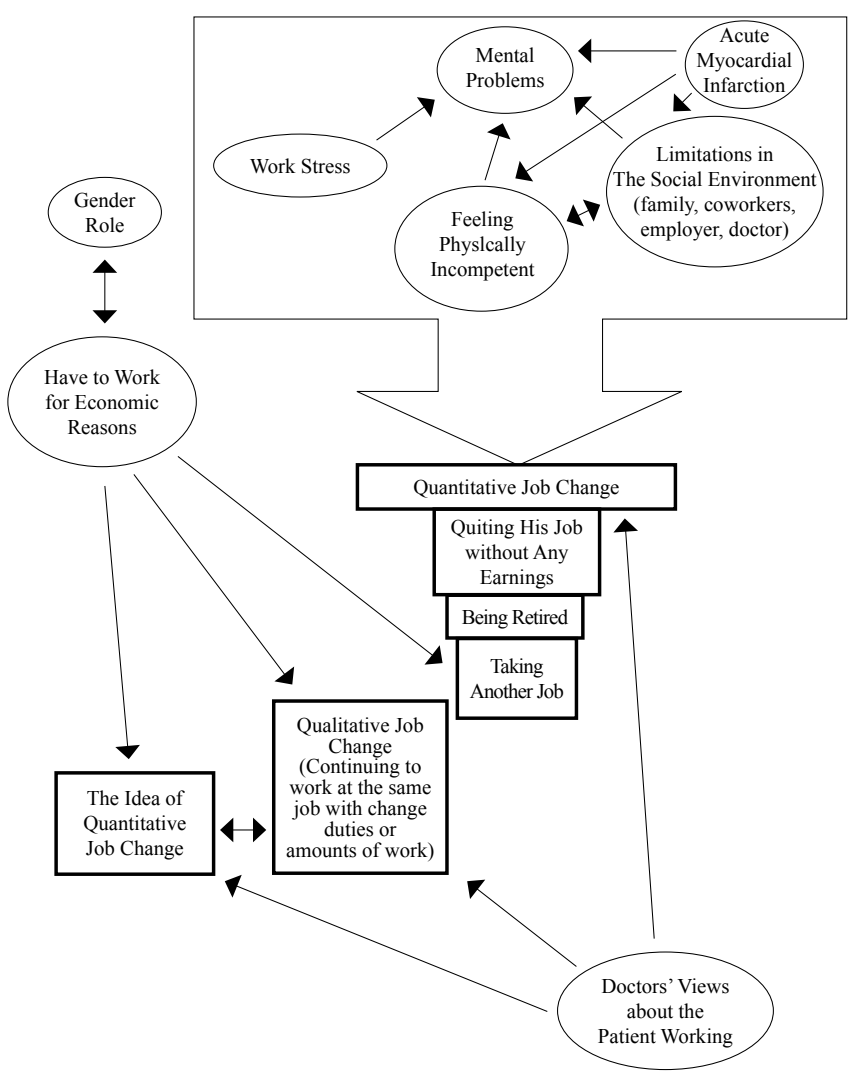

FIG. 1. Conseptual framework of the themes and reasons used in the study 


\section{Barriers of returning to work after AMI}

Except for one person, all of the participants said that they work less because of restrictions by social circles such as family, friends, and employers, or self-imposed restrictions. An employer of a patient told him to take care of himself, not to work too hard and to rest when he feels uncomfortable. On the other hand, some patients feel that they have been excluded from their work and social lives. Three participants said that they do not want their illness to be mentioned at the workplace and do not want to be reminded of it. A patient stated that he does not discuss his illness since he does not want to be treated differently. Patients who are working in paid jobs said that their jobs are not secure. So if someone has to be dismissed, they fear that it will be them because of their illness. A patient said "If a medical report exempts me from work again and again, my company may not accept that. Or they may hire another person to take my place".

Moreover, almost all the patients said that after the AMI they felt fearful of death, discouraged, and neglected. They stated that they wanted to enjoy social life, spend time with their families, make time for themselves and were less interested in their jobs.

Seven of the patients thought that they are so physically incompetent and inefficient that they want to move away from working life. A patient explained: "Even if I said that I'm unemployed and looking for a job, nobody will employ me. How he will benefit from me? I'm not productive. I can't give you anything. I'm a half person now. I got fired and couldn't find a job. I'm still waiting".

Half of the post-AMI patients said that they would get another job if they had the opportunity since they thought that their current jobs negatively affect their health. These statements reveal that some situations that existed prior to the diagnosis, such as stress at work, being unable to earn as much as they deserved, dissatisfaction, and work-home conflicts may have significantly affected the patients' subsequent working status. A patient said: "Before, I wasn't satisfied with my job. Since the illness I hate it". Patients often complained that they were unable to obtain sufficient support from the cardiologist or their workplace doctor after the illness. Most of the patients think that after the diagnosis doctors should offer more effective advice about their working status and lifestyle. A patient explained his expectations: "My doctor could have told me that my life would never be the same again. He could have told me what I should and should not do after the illness. He could have offered me guidance". Some doctors make restrictions which are not based on sufficient information about the working status of the patient and make comments which negatively affect the mental health of the patient. One of the patients said that he felt fearful due to doctor's attitude: "The doctor told me that I could die at any moment if I do not take care of myself. That was rough since I was newly discharged from hospital. It would have been better if he had told me gradually. My mood had been much better when I had been discharged. However, after I saw the doctor, I started to be afraid. He made me feel down. I never saw that doctor again, anyway."

\section{DISCUSSION}

In this study it has been revealed that while returning to work was high, most of the patients were starting to work less at the same job and some of them would like to find an easier job after AMI. Furthermore, we found that changes in working status after AMI have a variety of reasons including social and economic status in particular. Most of the patients continued to work due to their poor economic conditions and gender role. Since the participants are male, they said that they feel obliged to continue to work. It is also important that after AMI, they felt ambiguity about continuing to work and were unable to obtain sufficient support from their doctors regarding their working status.

The relationship between return to work and poor economic conditions has been reported as our study by previous studies. A study on patients who suffer from chronic back pain found that they still continue to work in spite of their pain since they fear losing their job and economic wellbeing (11). Similarly, Slebus et al. (12) claimed that economic problems also contribute to making people return to their jobs after AMI. Furthermore, after the diagnosis, spending increases due to current illness expenditures and earnings decrease since patients are unable to work at all or work for fewer hours. Patients may have to cope with economic problems while they try to enhance their health. To avoid these kind of situations, patients who are eligible to work should be encouraged to return to their jobs and work in suitable jobs.

Concerns following the CHD diagnosis are also related to gender roles. While men fear that they will fall behind at their jobs, women have difficulty fulfilling their responsibilities at home due to medical complications (13). Since men's earning are usually the main source of the family income, men are more likely to return to work than women (14). The patients in this study said that their main reason for returning to work was that they have to look after their families' economic wellbeing. Although this study produced no findings about female gender roles, since all of its participants were males, the effects of male gender attitudes on returning to work after the illness were strong. It is important to analyze the effects of social gender roles in different social classes and cultures. In societies where more women have jobs, the gender attitudes about returning to work after illness may vary. 
This study found that the patients' decisions to continue working was affected by their families', friends', and employers' suggestions, which may be restricting them from working. Some patients do not want their illness to be mentioned in their social environments, and one of the patients has hidden his illness from his colleagues. This could be considered social stigmatization. Stigma has been described as 'the situation of the individual who is disqualified from full social acceptance' (15). There are many studies about chronic diseases leading to social stigmatization (16). It would be instructive to conduct studies analyzing stigmatization. It is critical to give detailed information both to patients and their relatives about their competencies, daily activities, and work life. One study found that social support after diagnosis with CHD has positive effects on healthy habits, handling stressful situations and medication compliance. Therefore, the family and the social surroundings at the workplace should be instructed on how to behave and attempt to give the patients their lives back (17).

Our study shows that after AMI, patients may suffer from mental problems such as depression, anxiety, fear of death and discouragement. They may also feel physically incompetent. Some other studies suggest that depressed moods, heart failure, arrhythmia, comorbidity or physical incompetence after AMI reduce the percentage of people who return their jobs $(7,12,18-20)$. Returning to work mostly depends on the decision of the patient (7). Therefore, along with mental and physical problems, how patients see their health is also important. Many studies suggest that patients who perceive their health as poor are more likely to retire early or not return to their jobs $(19,21)$. If the patient does not have complaints about their illness, feels good, gets support from their colleagues and is provided with customized modifications at the workplace, it makes returning to the job easy. However, poor motivation and the job's negative effects dissuade people from returning to their jobs (12).

Some of the patients who were not satisfied with their working conditions said that they are less likely to tolerate them and therefore work less, while high work control and fairness in the workplace after CHD increase the likelihood of patients returning to their jobs, and high work expectations reduce it $(22,23)$. According to one study, high stress levels at work increase disability retirements after AMI, independent of the prevalence of the disease, psychological status and perceived health level (24). If patients are able get a paid leave of absence after AMI, then the percentage who return to work will increase significantly. Most patients think that flexible programs with less working hours would also increase the likelihood of patients returning to work (25). There is evidence that when flexible working conditions increase the control and preferences of employees so that they can prepare their work plans or retire partially, it improves their health and wellbeing. However, it is also known that flexible programs controlled by the employer, such as fixed-time contracts and part-time work, have negative effects on employee's health (26).

Although it is known that doctors' advice about patients' working status facilitates their return to their jobs, most of the patients stated that they were unable to obtain such advice similar to our study findings (12). According to a study, after adjusting for age, sex, and type of intervention, only doctor's advice remained strongly associated with return to work (HR: 47.6, 95\% CI 4.7-500). This shows that it is important that doctors use their experience to make decisions while considering the physical, sociodemographic, and psychological features of the patient (27). Using the cardiowork protocol, which suggests cooperation between the cardiologist and the workplace physician, makes it easier to return to work (28). Some studies offer advice about how doctors should help patients to return their jobs; however, the health system lacks sufficient legal regulations about this issue (29). Most doctors either make no comment about the working status of their patients, or they merely give subjective advice based on their own experience. Taking work conditions into consideration along with the patients' clinical and social situation, the support they need should be provided. Insensitive advice from doctors, given without considering their social lives and working conditions, can affect them negatively. Moreover, analyzing all of the factors in patient's life holistically and increasing medication compliance with monitoring can also be helpful.

Qualitative research allows for the subject to be understood through its detail, rather than analyzing causality in a specific domain. It is important to note that qualitative study features were strictly followed in this study e.g. face to face interviews with the patients in a quiet place, using semi-structured questionnaires, recording and note taking by the researcher and independent data analysis performed by three researchers. It is crucial to note that this study is the first of its kind in Turkey. Its limitations include some patients refusing to participate in the study, which meant that the number of cases was limited to 12 male patients. Interviewing more patients, including females, and conducting this study in different types of hospitals might provide more comprehensive information on returning to work after AMI.

Specific algorithms are needed and should be developed with the collaboration of different parties including cardiologists, workplace physicians, occupational safety specialists, employers and employees to increase the workability and return to work after illness. It may be possible to keep chronic patients on the job by giving them tailored work plans during the return period, suitable jobs, elaborated workloads and modified duties (30). Doctors do not have a guide book of 
objective advice about their patients' work status. To generate such advice, detailed studies, including country-specific evaluations to the conditions, such as those in Turkey, should be conducted. Doctors should learn to take an interest in the working lives of their patients, and appropriate in-service training should be provided for them. Along with well-designed social security policies, these patients should be supported by providing a decent work instead of exclusive approaches.

Ethics Committee Approval: Ethics committee approval was received for this study from the ethics committee of Dokuz Eylül University.

Informed Consent: Written informed consent was obtained from patients who participated in this study.

Peer-review: Externally peer-reviewed.

Author contributions: Concept - C.Ş., Y.D., Ö.A; Design - C.Ş., Y.D., B.K.; Supervision - Y.D., B.K., Ö.A.; Resource - Y.D., B.K., Ö.A.; Materials - C.Ş., Y.D., B.K.; Data Collection and/or Processing - C.Ş., Y.D., B.K.; Analysis and/or Interpretation - C.Ş., Y.D., B.K.; Literature Search - C.Ş., Y.D., B.K.; Writing - C.Ş., Y.D., B.K., Ö.A.; Critical Reviews - C.Ş., Y.D.

Conflict of Interest: No conflict of interest was declared by the authors.

Financial Disclosure: The authors declared that this study has received no financial support.

\section{REFERENCES}

1. Türkiye Hastalık Yükü Çalışması 2004. T.C. Sağlık Bakanlığı Refik Saydam Hıfzısıhha Merkezi Başkanlığı, Hıfzısıhha Mektebi Müdürlüğü. 1.Bask1. Ankara: Aydoğdu Ofset; 2007:1-12.

2. Wagner A, Arveiler D, Ruidavets JB, Bingham A, Montaye M, Ferrieres $\mathrm{J}$, et al. Gender- and age-specific trends in coronary heart disease mortality in France from 2000 to 2007: results from the MONICA registers. Eur J Prev Cardiol 2014;21:11722. [Crossref]

3. Alavinia SM, Burdorf A. Unemployment and retirement and illhealth: a cross-sectional analysis across European countries. Int Arch Occup Environ Health 2008;82:39-45. [Crossref]

4. Black D. Working for a healthier tomorrow 2008 [09.03.2015]. Available from: https:/www.gov.uk/government/uploads/system/uploads/attachment_data/file/209782/hwwb-working-fora-healthier-tomorrow.pdf.

5. Durmaz T, Özdemir Ö, Özdemir B, Keleş T, Bayram N, Bozkurt E. Factors affecting quality of life in patients with coronary heart disease. Turk J Med Sci 2009;39:343-51.
6. Bradshaw PJ, Jamrozik K, Gilfillan IS, Thompson PL. Return toWork After Coronary Artery Bypass Surgery in a Population of Long-Term Survivors. Heart Lung Circ 2005;14:191-6. [Crossref]

7. Mirmohammadi SJ, Sadr-Bafghi SM, Mehrparvar AH, Gharavi M, Davari MH, Bahaloo M, et al. Evaluation of the return to work and its duration after myocardial infarction. ARYA atheroscler 2014;10:137-40.

8. Mark D, Lam L, Lee K, Clapp-Channing N, Williams R, Pryor $\mathrm{D}$, et al. Identification of patients with coronary disease at high risk for loss of employment. A prospective validation study. Circulation 1992;86:1485-94. [Crossref]

9. Dekkers-Sánchez P, Wind H, Sluiter J, Frings-Dresen M. A qualitative study of perpetuating factors for long-term sick leave and promoting factors for return to work: Chronic work disabled patients in their own words. J Rehabil Med 2010;42:544-52. [Crossref]

10. Sverke M, Hellgren J, Naswall K. Job insecurity: A literature review. Stockholm, Sweden: National Institute for Working Life, 2006.

11. De Souza L, Frank A. Patients' experiences of the impact of chronic back pain on family life and work. Disabil Rehabil 2011;33:310-8. [Crossref]

12. Slebus FG, Jorstad HT, Peters RJ, Kuijer PP, Willems JH, Sluiter JK, et al. Return to work after an acute coronary syndrome: patients' perspective. Saf Health Work 2012;3:117-22. [Crossref]

13. Emslie C. Women, men and coronary heart disease: a review of the qualitative literature. $J$ Adv Nurs 2005;51:382-95. [Crossref]

14. King KM. Gender and short-term recovery from cardiac surgery. Nursing Res 2000;49:29-36. [Crossref]

15. Goffman E. Stigma: Notes on the Management of Spoiled Identity. 1963.

16. Lee SM, Lim LC, Koh D. Stigma among workers attending a hospital specialist diabetes clinic. Occup Med 2015;65:67-71. [Crossref]

17. Boutin-Foster C. Getting to the heart of social support: a qualitative analysis of the types of instrumental support that are most helpful in motivating cardiac risk factor modification. Heart Lung 2005;34:22-9. [Crossref]

18. Bhattacharyya MR, Perkins-Porras L, Whitehead DL, Steptoe A. Psychological and clinical predictors of return to work after acute coronary syndrome. Eur Heart J 2007;28:160-5. [Crossref]

19. Brink E, Brandstrom Y, Cliffordsson C, Herlitz J, Karlson BW. Illness consequences after myocardial infarction: problems with physical functioning and return to work. $J$ Adv Nurs 2008;64:587-94. [Crossref]

20. McBurney CR, Eagle KA, Kline-Rogers EM, Cooper JV, Smith DE, Erickson SR. Work-related outcomes after a myocardial infarction. Pharmacotherapy 2004;24:1515-23. [Crossref] 
21. Kiessling A, Henriksson P. Perceived cognitive function in coronary artery disease--an unrecognised predictor of unemployment. Qual Life Res 2005;14:1481-8. [Crossref]

22. Du CL, Cheng Y, Hwang JJ, Chen SY, Su TC. Workplace justice and psychosocial work hazards in association with return to work in male workers with coronary heart diseases: a prospective study. Int J Cardiol 2013;166:745-7. [Crossref]

23. Slebus FG, Kuijer PP, Willems JH, Sluiter JK, Frings-Dresen MH. Prognostic factors for work ability in sicklisted employees with chronic diseases. Occup Environ Med 2007;64:814-9. [Crossref]

24. Laine S, Gimeno D, Virtanen M, Oksanen T, Vahtera J, Elovainio M, et al. Job strain as a predictor of disability pension: the Finnish Public Sector Study. J Epidemiol Community Health 2009;63:24-30. [Crossref]

25. Earle A, Ayanian JZ, Heymann J. Work Resumption after Newly Diagnosed Coronary Heart Disease: Findings on the Importance of Paid Leave. J Womens Health (Larchmt) 2006;15:4. [Crossref]
26. Joyce K, Pabayo R, Critchley J, Bambra C. Flexible working conditions and their effects on employee health and wellbeing (Review). Cochrane Database Syst Rev 2010; CD008009. [Crossref]

27. Farkaš J, Černe K, Lainščak M, Keber I. Return to work after acute myocardial infarction-Listen to your doctor. Int J Cardiol 2008;130:14-6. [Crossref]

28. Scafa F, Calsamiglia G, Tonini S, Lumelli D, Lanfranco A, Gentile E, et al. Return to work after coronary angioplasty or heart surgery: a 5-year experience with the "CardioWork" protocol. $J$ Occup Environ Med 2012;54:1545-9. [Crossref]

29. DeBusk R, Dennis C. Occupational Work Evaluation of Patient with Cardiac Disease: A Guide for Physicians. West J Med 1982;137:515-20.

30. Shrey DE, Mital A. Accelerating the return to work (RTW) chances of coronary heart disease (CHD) patients: part 2--development and validation of a vocational rehabilitation programme. Disabil Rehabil 2000;22:621-6. [Crossref] 